Revue d'histoire de l'Amérique française

REVUE D.HISTOIRE DE L'AMÉRIQUE FRANÇAISE

\title{
James Cuthbert, père, et ses biographes
}

\section{Edouard Fabre-Surveyer}

Volume 4, numéro 1, juin 1950

URI : https://id.erudit.org/iderudit/801617ar

DOI : https://doi.org/10.7202/801617ar

Aller au sommaire du numéro

Éditeur(s)

Institut d'histoire de l'Amérique française

ISSN

0035-2357 (imprimé)

1492-1383 (numérique)

Découvrir la revue

Citer cet article

Fabre-Surveyer, E. (1950). James Cuthbert, père, et ses biographes. Revue

d'histoire de l'Amérique française, 4(1), 74-89. https://doi.org/10.7202/801617ar d'utilisation que vous pouvez consulter en ligne.

https://apropos.erudit.org/fr/usagers/politique-dutilisation/ 


\section{JAMES CUTHBERT, PERE, ET SES BIOGRAPHES}

James Cuthbert, premier seigneur de Berthier, est mort le 17 septembre 1798. D'après les désirs exprimés dans son testament, sa succession devait être finalement liquidée cent cinquante ans après sa mort, c'est-à-dire en 1948. Hélas! l'homme propose, et rien de plus. De ses vastes domaines terriens, il ne reste guère que le manoir Dautray, entre Berthier et Lanoraie, qui soit en la possession d'un descendant sans postérité. J'avais songé à commémorer cet anniversaire par une biographie de James Cuthbert, mais il me manquait trop de détails. L'année qui s'est écoulée depuis m'a apporté peu de renseignements, et je livre mon travail tel quel, laissant à d'autres le soin de le compléter et de le corriger, s'il y a lieu.

James Cuthbert, père, seigneur de Berthier et autres lieux, a eu ses biographes: D.R. McCord, Q.C., l'abbé S.-A. Moreau, l'historien de Berthier, le regretté Francis-J. Audet, M. Régis Roy, M. le notaire Richard Lessard, de Sainte-Ursule, - j'en oublie peut-être, - sans parler des dictionnaires biographiques du R. Père Lejeune, de W.S. Wallace et de Gustave Turcotte: Conseillers législatifs.

Après avoir parcouru toutes ces études, j'ai dâ constater que les matériaux manquaient encore pour une biographie définitive, et j'ai abandonné mon projet, sauf à relever quelques erreurs et à combler quelques lacunes.

Quels sont les éléments essentiels d'une biographie? Assurément, ceux qui sont consignés dans des actes de l'état civil: la naissance, le mariage (si mariage il y a eu) et la mort.

Quant aux mariages, Cuthbert en eut trois. Du premier, l'auteur le plus précis sur la question, M. Audet, nous dit: 'L'honorable James Cuthbert avait épousé, en Écosse, Margaret, fille de Sir Roderick MacKenzie et de Janet Grant"... Il ne s'accorde pas avec le Scots 
Ancestry Research Society. D'après cette dernière, ce serait Hugh Cuthbert qui aurait fait ce mariage, le 18 juin 1748, alors que James aurait, le 16 novembre 1742, épousé Marjory Anderson. Nous n'avons pas trace de la mort de Marjory Anderson.

M. Audet continue: "Ayant divorcé sa première femme, il en épousa une autre au Canada, en 1766, et en eut trois fils et sept filles." Nous n'avons aucune preuve de ce divorce, qui était chose à peu près inconnue en Écosse à cette époque. Nous n'avons nulle trace de la mort de la première madame Cuthbert.

Sa deuxième femme se nommait Catherine Cairns. Elle était la fille de David Cairns et de Catherine Wilson, et sœur de madame Donald Morrison, de Montréal. Elle mourut le 7 mars 1785, âgée de quarante ans (donc née en 1745). Alexander Cairns, son frère, fut agent de la seigneurie, à Berthier. Sa sœur, madame Donald Morrison, mourut à Berthier le 25 mars 1825, âgée de 72 ans, catholique.

C'est encore incomplet. Mgr Tanguay qui nous parle de ce mariage (protestant), ne nous en donne ni la date, ni le lieu, pas plus qu'il ne nous dit où était née Catherine Cairns.

Enfin, Cuthbert se maria une troisième fois. Cette fois, nous avons la date du mariage. Voici ce qu'on trouve dans le registre de l'église protestante des Trois-Rivières:

"1786. Le vingt et trois de mars après avoir obtenu la licence du Commandant en chef, Colonel Henry Hope, j'ai marié James Cuthbert, seigneur de Berthier, et Rebecca Cochrane, et ce en présence de plusieurs témoins."

Il n'y a pas de signatures, sauf celle du pasteur Veyssière. S'il y en avait eu, on aurait su que la mariée ne s'appelait pas Cochrane, mais Stockton. C'était la plus jeune des filles de John Stockton, Écuier, juge de la cour des plaids communs du comté de Somers, New Jersey. On découvre le fait dans le testament olographe de Cuthbert, déposé à Montréal. Chose bizarre, Cuthbert ne la désigne pas comme sa femme, mais l'appelle "Rebecca Stockton, otherwise called Rebecca Cuthbert". Pour savoir qu'elle était bien sa femme, il faut avoir recours à une procuration passée le 14 octobre 1795, devant de Glandon, notaire, où sa qualité est indiquée. 
Avant de convoler une troisième fois, Cuthbert avait passé contrat devant le notaire Faribault, qui prénomme la mariée Phebena, au lieu de Rebecca ${ }^{1}$.

C'est le seul contrat de mariage que j'aie pu retracer jusqu'à présent, ce qui est étrange. Que son premier mariage, qui a eu lieu en Écosse, n'ait pas été précédé d'un contrat, c'est admissible. Mais en 1766, il était déjà propriétaire de la seigneurie de Berthier, qu'il avait payée 70,000 livres, plus une rente, et dont les revenus, au moins, entraient en communauté. Durant son mariage avec Catherine Cairns, il acquit les seigneuries Du Sablé, de l'Ormier, Dautray, Lanoraie et Maskinongé (partie), et du vivant de Rebecca Stockton, le fief d'Orvilliers. Son testament n'est pas celui d'un homme commun en biens. Cuthbert a signé des centaines d'actes notariés: en on trouve dans cinq ou six greffes. Son fils James, qui s'est marié deux fois, a, chaque fois, passé un contrat de mariage. Son fils Ross a aussi passé contrat. Il serait bien étonnant que leur père n'en eât pas fait autant.

Enfin, la date de sa mort n'a pas encore été publiée jusqu'ici. Il est mort à Berthier le 17 septembre 1798 et a été inhumé le lendemain. Son testament, sous la forme anglaise, en date du 4 aout 1798, est un document précieux au point de vue de sa généalogie. Les actes de l'état civil d'un homme sont peu de chose auprès de ce qu'il a accompli.

Le 24 janvier 1778, était signé le document suivant, qui fut remis a James Cuthbert et dont le petits-fils, Edward Octavian, a donné communication à M. l'abbé Moreau, l'historien de Berthier:

A tous et a chacun de ceux que les présentes concernent ou pourront concerner, nous, John Hooke Campbell de Bangeston, Ecuyer, horaut d'armes du Lyon, certifions et déclarons par les présenties que les Insignes et Armoiries se rapportant et appartenant a l'Honorable James Cuthbert, Ecuyer, propriétaire des fiefs ou seigneuries de Berthier, Maskinongé, New York, autrement appelé Dusablé, Dautray, Lanoraie et de l'Ormier, dans le district de Montréal et la province de Québec, Amérique du Nord, l'un des conseillers législatifs de sa majesté dans la dite province, et ci-devant

1. Nous devons ce renseignement et beaucoup d'autres, à Madame Florence Fornet-Martel que nous remercions. 
capitaine dans le quinzième régiment d'infanterie, fils et héritier d'Alexandre Cuthbert, d'Inverness, Ecuyer, et de Béatrix, fille de David Cuthbert, d'Ardresie, Ecuyer; lequel Alexandre était fils et héritier de Jean Cuthbert, d'Inverness, Ecuyer, et de Marie, fille de ...MacLean, de Dachgarrich, Ecuyer; lequel Jean était le second fils de Jean Cuthbert,baron de Castlehill, chef de cet ancien nom de Sir, et de Marie, fille de George Cuthbert, de Castlehill,Ecuyer, arrièrepetit-fils de Guillaume Cuthbert, de Castlehill, Ecuyer, qui était fils et héritier de Jean Cuthbert, Ecuyer, qui était fils et héritier de Jean Cuthbert, de Castlehill, Ecuyer, qui commanda les Forces levées par la ville d'Inverness à la bataille de Harlane, en l'an 1411, contre le rebelle Donald, seigneur des Isles, dont il a saisi le drapeau; lequel dernier George était le représentant d'une ancienne famille saxonne de ce nom dans le royaume de Northumberland; sont immatriculés dans les registres publics du bureau du Lyon, et se blasonnent comme à la marge de la manière suivante, savoir: D'or à la fasce d'azur chargée de trois fraisiers d'argent. Au chef chargé d'une bisse s'élevant du second en pal, armé et langué de gueules. Au-dessus de l'écu est placé un heaume en rapport avec son degré (baron), bordé de gueules et double d'argent. Sur une guirlande de ses livrées, et comme cimier, une dextrochère armée sortant en pal de la guirlande et tenant une flèche propre. Et sur une oriflamme, au-dessus du cimier, cette devise "Fortier", lesquels insignes et armoiries ci-dessus blasonnés, nous, par les présentes, ratifions, confirmons et assignons au dit honorable James Cuthbert, écuyer, et aux héritiers de son corps comme leurs propres livrées et armoiries dans tout temps à venir.

En foi de quoi les présentes ont été souscrites par Robert Boswell, écuyer, notre député, et le grand sceau de notre bureau (datant de 1673), y a été déposé à Edinbourg, le vingt quatrième jour de janvier de l'an mil sept cent soixante dix huit.

R. Boswell

Lyon Dep. ${ }^{2}$

Dans "Representative Canadians", publié en 1891, il est dit ce qui suit (j'emprunte à l'abbé S.-A. Moreau, dans son Histoire de Berthier, p. 38):

L'Honorable Jacques Cuthbert, père, servit d'abord comme lieutenant dans la marine anglaise, et se trouvait sur le vaisseau amiral au bombardement de Carthagène, où il fut chargé de porter en

2. La traduction est de l'abbé Moreau; les mots entre parenthèses sont de nous. 
Angleterre les dépouilles de cette forteresse. Sorti bientôt de la marine, il fut nommé commandant d'une compagnie des milices indépendantes formées à Inverness, lesquelles s'appelaient d'abord la Garde Noire et sont aujourd'hui connues sous le nom du $42^{\text {eme }}$ Ecossais, et il fit quelque temps de service dans ce corps.

Il entra ensuite au $15^{\circ}$ régiment dans lequel il devint capitaine. Il était en cette qualité présent à la prise de Louisbourg et à la Bataille des Plaines d'Abraham, en 1759, où il était aide de camp du général Wolfe. C'est lui qui fut choisi par le général Murray, pour porter en Angleterre la nouvelle de la prise de Québec.

David R. McCord, dans le Dominion Illustrated News de 1891, donne les mêmes faits, avec quelques précisions. M. F.-J. Audet, parlant de la mission de Cuthbert après la chute de Québec, dit en note: "Nous n'avons pu obtenir confirmation de ce fait." Même lorsque M. Audet écrivait, en 1935, M. Régis Roy avait déjà dénié cette assertion en s'appuyant sur Burke's peerage, l'almanach de Gotha anglais, où il est dit:

Sir James Douglas, a naval officer of eminence... was knighted in consideration of bearing home the dispatch announcing the surrender of Quebec.

D'autre part, M. le notaire Richard Lessard, de Sainte-Ursule, s'appuyant sur des textes extraits des papiers Northcliffe, dit que cette nouvelle fut donnée par un colonel Hale.

Je laisse saint Thomas s'accorder avec Scot, mais, sans dire que l'un ou l'autre a raison, je suis prêt à admettre que ce n'est pas Cuthbert qui a transporté la nouvelle, quoi qu'on en ait dit.

En mars 1796, il était en Angleterre, où il demandait au duc de Portland, entre autres choses, de faire de lui un baronet, ce qui, disait-il, lui avait été offert par Lord Garmain, en 1776, pour ses actions d'éclat pendant l'invasion américaine, aux Trois-Rivières. En décembre de la même année, il réitère sa demande, rappelant qu'en avril 1776, il aurait, au péril de sa vie, sauvé le brigadier Fraser avec 700 à 800 membres de son armée et sept vaisseaux. Il parle de son emprisonnement par les Américains, de ses pertes d'argent, des situations qu'il a occupées, et déclare: "Sixty years have silvered me in its constant service both by sea and land." Croit-on que s'il eat fait quelque 
action d'éclat, so 't à Carthagène ou à Québec, il n'y aurait pas fait la moindre allusion? Suivant Burke's peerage, celui qui a apporté à Londres la nouvelle de la prise de Québec a reçu un titre de noblesse. Si c'eut été Cuthbert, aurait-il négligé de rappeler sa conduite après Québec, sinon après Carthagène?

Il en est de même de Louisbourg. Y était-il, ou l'a-t-on confondu avec son homonyme, John Cuthbert, fils de John Cuthbert, de Castlehill, officier qui y fut tue le 25 juin 1758 , alors qu'il combattait sous Wolfe? Je crois qu'il y a eu confusion.

En constatant le désaccord entre les historiens de Cuthbert, et la difficulté de trouver des preuves sérieuses de sa conduite à Carthagène et à Louisbourg, - sa conduite à Québec étant déniée, - j'ai renoncé à me faire le biographe de James Cuthbert, père. Notons seulement ces faits rapportés par ses biographes, qu'en 1766, il fonda, pour ainsi dire, la paroisse de Saint-Cuthbert, en donnant pour y bâtir une église, soixante arpents de terre, deux cloches et un tableau de saint Cuthbert, à la seule condition que l'église portât son nom ${ }^{3}$. Cuthbert prêta serment comme Conseiller législatif le 14 juin 1766. Il y siégeait encore le 24 aout $1775^{4}$. Enfin, en 1792, il fut candidat au poste de député de Warwick (c'était le nom du comté dont Berthier était le chef-lieu) contre Pierre Paul Margane de Lavaltrie, dont il tenta en vain de faire annuler l'élection, en prétendant qu'il n'était pas sujet britannique, prétention qui ne fut pas acceptée par les autorités ${ }^{5}$. Il eut aussi des démêlés avec le curé Pouget, de Berthier.

En attendant, j'avais cherché à me renseigner sur sa postérité.

Sur ce point, nous avons la déclaration de Cuthbert quant au nombre de ses enfants, grâce à l'inscription qu'il a fait placer dans la chapelle qu'il a construite, à Berthier. Il y dit que Catherine Cuthbert, son épouse, décédée le 7 mars 1785 , était mère de trois fils et de sept.

3. Abbé S.-A. Moreau, (Berthier 1889), 142.

4. Gustave Turcotte, Le conseil législatif de Québec (Beauceville, 1933), 27; Documents constitutionnels 1759-1791, 249, 251, 684, 703, 706.

5. F.-J. Audet et E. Fabre-Surveyer, Les deputés au premier parlement du BasCanada (1792-17 6), (Les éditions des Dix, Montréal, 1946), t. I: 311-12. 
filles, et qu'ils avaient été mariés 19 ans, ce qui porte à 1766 l'année de son mariage, dont nous ignorons la date et le lieu.

“Sept filles, dit M. Audet, s'il n'y en a pas eu neuf ou dix!" Nous verrons pourquoi $M$. Audet exprime ce doute, du reste mal fondé. Voici la liste de ces enfants, qui sont, j'imagine, tous nés à Berthier:

1. $A L E X A N D E R$, né le 14 aout 1767 , baptisé a Montréal le 29 du même mois, fut envoyé, ainsi que ses frères, faire ses études au collège catholique de Douai, en Flandre, où l'on recevait surtout des élèves d'Angleterre, d'Écosse et d'Irlande. D'après l'abbé Moreau, leur père leur aurait dit, à propos de la religion catholique: "Pratique cette religion, et quand tu seras en majorité, tu choisiras celle que tu préféreras". Et, ajoute-t-il, Alexander aurait déclaré par Ǵcrit: "Laquelle choix j'ai fait. En foi de quoi, à Berthier, le 2 mars 1790". M. l'abbé Moreau en conclut qu'Alexander et Jacques estimaient assez le catholicisme pour s'y attacher et y mourir. M. Audet dit qu'Alexander "embrassa la religion catholique qu'il pratiqua toute sa vie."

Ces messieurs ont-ils pris connaissance du document suivant, copié des registres du Christ Church, de Sorel:

Lord's Day, 17th September 1797.

On the fourteenth day of September, Anno Domini one thousand seven hundred and ninety seven, personally appeared in the Chancel of this Church Alexander Cuthbert, of the parish of Berthier, and then and there in the presence of the subscribing witnesses, made his solemn Declaration against Popery, according to the $30 \mathrm{C} 2$, St. 2, c. 1 , and in the very words therein prescribed and appointed to be in such cases read and repeated.

Alex ${ }^{r}$ Cuthbert. - Witnesses

John Doty, Rector, Wm Nelson, Johan Daniel Dirge, warden."

J'ai cherché en vain l'acte de décès d'Alexander Cuthbert. On sait qu'il mourut le 9 janvier 1810 et qu'il fut enterré dans la chapelle Cuthbert, presbytérienne, où l'on ne tenait pas de registres.

Etait-il marié? McCord dit qu'il se maria mais ne laissa pas 
d'enfants. C'est plutôt le contraire qui est vrai, car le 30 avril 1792 , Cuthbert, père, faisait une donation à Marie dite Maritaine, fillette âgée de 3 ou 4 ans, que l'on dit être issue des œuvres d'Alexander Cuthbert, son fils, avec Marie Jeanne Serin, ci-devant de Montréal.

En somme, Alexander Cuthbert ne parait pas avoir jeté un grand lustre sur la famille. Il vécut à Dautray, où il se trouvait en janvier 1804, quand le manoir devint la proie des flammes. Il reçut, par le testament de son père, les revenus de la seigneurie de New-York (Dusablé), du fief de Saint-Jacques, ou Chenail du Nord, et de la partie des fiefs Maskinongé et Lornier. Il fut plusieurs fois parrain des enfants de ses parents.

2. JAMES, né le 4 juin 1769 , baptisé le 29 , à Montréal. Nous en parlerons plus tard.

3. Ici, M. Audet place $B E A T R I X$ Cuthbert, née le 22 novembre 1770 , baptisée le 11 janvier 1771 , et qui aurait épousé le capitaine Clark. Ce prénom de Beatrix, bien approprié puisque c'est celui de la mère du seigneur Cuthbert, a été pris de la compilation des actes de l'état civil de l'église du Christ, de Montréal, publiée dans le rapport des Archives du Canada de 1885. En réalité, celle qui naquit le 22 novembre 1770, reçut au baptême, le 15 janvier 1771 , le prénom de Catherine, et fut appelée par son père, dans son testament, Catherine Betty Isabella. En 1792, elle épousa John Antrobus, épicier à Québec, puis à Berthier. Le 18 décembre 1792, les époux habitaient à Berthier une villa appelee Northfield, et Cuthbert leur faisait donation, que le notaire Joran qualifie de don gratuit, d'une portion de terre d'un demi-arpent de front sur $\mathbf{4 0}$ de profondeur, située au Chenail du Nord et bornée au front par le fleuve Saint-Laurent, à charge par les donataires de payer au donateur, tous les 11 novembre, fête de SaintMartin, une rente en argent et en blé. Le 11 juin suivant, Antrobus était nommé grand voyer pour le district de Trois-Rivières. Leur fils ainé, Edouard William Romer Antrobus, nommé d'après son parrain le lieutenant colonel J.W.A. Romer, eut une carrière remarquable (B.R.H., 1906, p. 78 et seq.). Ils eurent d'autres enfants, dit la Gazette de Québec du 4 mars 1815. Madame Antrobus mourut le 22 janvier 1806 et fut inhumée le 24 par le pasteur Rudd, de l'église du Christ, de 
Sorel. Son corps repose dans la chapelle de Berthier. Antrobus mourut aux Trois-Rivières le 8 mai $1820^{\circ}$.

4. MARY ANN. Nous n'avons pas la date de sa naissance, mais son père, dans la donation qu'il lui fit, à elle et à son mari, le 20 mai 1794, la déclare la deuxième de ses filles. Elle était majeure quand elle épousa, le 7 avril 1794, à la maison de campagne de son père, à Beauport, John William Augustus Romer, major au premier bataillon du $60^{\circ}$ régiment. Nous leur connaissons deux filles: Caroline Ann Augusta, née le 19 juin 1795 et baptisée le 7 aout suivant, et Georgina Clara Lavinia, née le 22 janvier 1797 et baptisée le 7 février.

Romer, probablement natif d'Angleterre, parvint, dans l'armée, au rang de brigadier. En 1795, il annonçait à louer sa maison de la rue du Palais, à Québec, et, en qualité de lieutenant-colonel, prenait le commandement du fort de l'Ile-aux-Noix. En 1798, il était colonel et passait au $60^{\circ}$ régiment (Wiltshire). De 1797 à 1807, il est aux Iles Sous-le-vent avec le rang de brigadier. En 1808, il est encore dans l'Army List, mais non dans la liste des brigadiers, après quoi nous le perdons de vue, ainsi que sa femme.

5. GEORGINA-DAVIDA, dont nous ignorons la date de naissance, mais que son père appelle sa troisième fille, et qui est déclarée "spinster" dans son acte de mariage, ce qui la ferait naître vers 1773 . Elle épousa, le $1^{\text {er }}$ janvier 1794, à Beauport, Georges-Louis-V ctor Forneret, fils de noble Albert Forneret, l'un des conseillers de l'honorable conseil de la ville de Lausanne, canton de Berne, en Suisse ${ }^{7}$. Lieutenant au $60^{\circ}$ régiment d'infanterie depuis le 9 novembre 1789 , capitaine le 16 décembre 1795, Forneret fut promu major le 24 avril 1808. Il était à la bataille de Corunna, le 16 janvier 1809 , sous les ordres de Sir John Moore. Son nom disparaît de l'Army List après 1810. D'après l'arbre généalogique de la famille, il serait mort à la Jamai̊que en 1821. Sa femme l'aurait prédécédé, étant morte en avril 1816.

6. Voir sa biographie par P.-G. Roy dans: Les Cahiers des Dix, t. 8 (Montréal, 1943): 231-232, et par F.-J. Audet, dans les Mémoires de la Sociele $R$ yale (1935), 147.

7. D'après l'arbre généalogique de la famille Forneret, il aurait été reconnu comme ancien hourgeois de Genève, le 4 octobre 1784, et serait mort le 14 mai 1787. 
Voici la liste de leurs enfants, d'après l'arbre généalogique de la famille, complété par nos propres recherches:

a) Cuthbert, né le 14 février 1796 (nous ne savons où), fut officier au $60^{\circ}$ régiment. Lieutenant, il fut mis à la demi-paye le 25 décembre 1818. Il épousa, le 18 février 1816, probablement à Berthier, FrançoiseCatherine Massey, qui mourut en Irlande le 24 juillet 1836 . Quant à lui, son nom disparaît de l'Army List en 1831.

b) Albert, né à Berthier en 1797, mort au même endroit en bas âge (non corroboré).

c) Edward Alexander, né à Berthier le 14 février 1798 et baptisé le 13 juin suivant. Il dut mourir jeune, car le 14 février 1804, on donnait à un autre fils le même prénom et le même parrain, Alexander Cuthbert, son oncle.

d) George Matthew, né le 13 avril 1800 et baptisé le 17 octobre suivant. Il serait mort à la Jamaïque.

e) Albert, né le 24 mai 1802, baptisé le 28 juin, inhumé le 10 juillet de la même année (prénommé Edward dans l'arbre généalogique).

f) Charles Alexander, né le 10 décembre 1803, baptisé le 14 février 1804. Admis à la profession de notaire, le 29 janvier 1828, il épousa, en premières noces, à Berthier, sans doute, Elizabeth Barbier, de Berthier, et eut d'elle cinq enfants, dont l'aîné, Mary, naquit le 18 juillet 1845, et le plus jeune, Emma, le 18 décembre 1852. Un fils, George Augustus, né le 23 septembre 1851, épousa, en juin 1888, Emma Adelaide Robbins, de Hamilton, Ontario. (Voir sa biographie dans Canadian Men and Women of the time (Morgan), 1898 et 1912, et, dans l'édition de 1898, celle de sa sœur Julia Elizabeth, diaconesse, qui vit encore). Forneret devint archidiacre. Leur fils George Reginald vit encore, ainsi qu'une fille, Marion Adelaide.

Le 16 octobre 1858, le notaire Forneret épousa:t Anny Kerrigan, dont il eut sept enfants. L'un d'eux, Victor F.W., né le 13 aout 1863, épousa à Montréal, le 23 décembre 1907, après contrat de mariage devant P. Tellier, notaire, Mary Bostwick, fille de John Bostwick, seigneur de Lanoraie, et d'Elizabeth Lloyd Merrick. Ingénieur civil, il était en charge du chenal du Saint-Laurent. Il mourut à Montréal 
le 19 avril 1932 et sa veuve, le 24 décembre 1946. Ils ne laissèrent pas d'enfants.

Le notaire Forneret mourut à Berthier le 10 mars 1880 .

Ici s'arrêtent nos recherches personnelles. D'après l'arbre généalogique, les enfants suivants, au moins, seraient nés en Angleterre:

g) Archibald, né le 11 septembre 1807, serait mort à la Jamaïque.

h) Georgiana Mary Ann, née en Angleterre le 25 décembre 1808, épousa à Berthier, le 3 septembre 1832, Edward Adams Clark, avocat à Montréal. Elle mourut à Berthier le 21 octobre 1845. Son mari épousa, en 1857, une cousine de sa femme, Georgina Cuthbert, veuve d'Augustus David Bostwick, des Trois-Rivières.

i) Frederick, né en Angleterre le 16 février 1812, y mourut le 6 juin 1816.

De toutes les filles de James Cuthbert, père, madame Forneret est celle dont on peut le plus facilement retracer la descendance. Le dernier descendant mâle de cette famille est le pasteur George Reginald Forneret, chapelain protestant du pénitencier de Saint-Vincentde-Paul, auquel nous devons le prêt de l'arbre généalogique. Comme il n'a pas d'héritier mâle, ce beau nom s'éteindra avec lui.

6. MARGARET ETHELIND, née le 29 février 1772 , baptisée à Montréal le 17 mai 1772, épousa à Berthier, le 8 juillet 1797, Thomas Clarke, capitaine au $60^{\circ}$ régiment d'infanterie (Royal Americán). Cuthbert leur fit une donation, le 6 juillet 1797. Nous ne savons pas encore si c'est ce Thomas Clarke que l'on retrouve en 1828 dans le Haut-Canada, avec le grade de colonel. Son nom disparait de l'Army List en 1807.

7. $J A N E$, née le 12 mars 1773 et baptisée à Montréal le 23, épousa à Berthier, le 3 mars 1796, Daniel Nixon, natîf de Congleton, Angleterre, lieutenant au $60^{\circ}$ régiment d'infanterie. Son nom disparait de l'Army List en 1809.

Le 24 novembre 1808, elle épousait en secondes noces le major Robertson, des "Canadian Fencibles", qui, nommé major le 20 avril 
1803, fut promu lieutenant-colonel le 27 juin 1811 et mis à la demisolde le 24 août 1816.

8. ROSS, né le 17 février 1776, baptisé le 28 à Montréal. Après ses études à Douai, il fit son droit à l'“Inner Temple", de Londres, fut admis au Barreau du Bas-Canada le 18 juin 1803 et exerça sa profession à Québec avec succès. Il était député du comté de Warwick (avec son frère James) depuis le 28 juillet 1800 . Il siégea jusqu'au $1^{\text {er }}$ mars 1810, puis du 11 aoât 1812 au 29 février 1816, et enfin du 11 avril au 29 mai 1820. Il épousa Emily Rush, fille du docteur Benjamin Rush, de Philadelphie, l'un des signataires de la déclaration de l'indépendance des Etats-Unis. Nous ignorons la date de leur mariage et du contrat qui l'a précédé, stipulant séparation de biens; mais le 20 octobre 1801, à Berthier, ils faisaient baptiser une fille, Julia, née le 2 du même mois, qui mourut le 11 février 1802 et fut inhumée le 13. Un fils, James, naquit à Berthier, en 1800 , et mourut en Crimée, d'une insolation, le 20 mars 1842, laissant un fils, Edmund Charles, seul survivant de quatre fils. James avait épousé, à Berthier, le 15 juin 1834, Jane Stephens, qui alla vivre en Angleterre.

Une fille de Ross Cuthbert, Mary, née le 25 février 1810, fut baptisée à l'église métropolitaine de Québec le 13 juillet 1810. Son père était alors juge de la Cour des Sessions de quartier. Elle mourut à Berthier, en faisant sa prière, parait-il, le 19 mars 1888 . Une autre fille, Georgina, née le 7 juillet 1803 et baptisée le 28, épousa en premières noces, à Lanoraie, le 24 juin 1829, Augustus David Bostwick, avocat aux Trois-Rivières, admis au Barreau en 1819, et collaborateur de Hughes Heney grand voyer.

Ils eurent cinq enfants, nés aux Trois-Rivières:

a) Ann Emily, née le 5 juin 1830, décédée au même endroit le 16 août 1831 ; b) Mary, née le 3 mars 1833, épousa, le $1^{\text {er }}$ décembre 1855, Edward Octavian Cuthbert, qui devint seigneur de Berthier et mourut en 1890; elle mourut le 6 février 1885; c) Georgina, née le 4 octobre 1835, épousa en avril 1855 le lieutenant-colonel James W. Hanson, qui décéda à Berthjer le 11 janvier 1911. Ils n'eurent pas d'enfants; d) Charles Ogden, né le 24 juillet 1836 et baptisé le 18 octobre suivant, dont nous ne savons rien de plus; et e) John, né le...? 
marié à Berthier le 22 novembre 1860 à Elizabeth Lloyd Merr ck, décédé à Berthier le 2 janvier 1907, et son épouse, le 15 du même mois. Ils eurent trois enfants, dont le plus jeune, Augustus John Ross, né en 1869, vit encore. Marié à Toronto le 4 mai 1907, à Margaret Macdonald Dickson, qui vit encore. Ils n'ont pas d'enfants.

Ross Cuthbert fut un homme remarquable, dont la biographie a été écrite par F.-J. Audet (op. cit., p. 143-5) et le notaire Richard Lessard. Il mourut à Lanoraie, âgé de 85 ans, le 28 avril 1861, et fut inhumé dans le cimetière protestant de Sorel. Sa femme était prédécédée, étant morte le 27 avril 1850.

9. $M A R Y$, ou Maria Clara, née le 15 septembre 1780, fut baptisée le 8 novembre suivant. Elle fut marraine, le 14 février 1804, de son neveu Charles Alexander Forneret, après quoi nous la perdons de vue.

10. CAROLINE. Tout ce que nous savons d'elle, c'est qu'elle fut inhumée dans la chapelle de Berthier, avant 1786. C'est à tort que M. Audet dit d'elle: "Elle était mariée et avait des enfants". L'erreur vient de la mauvaise rédaction de l'inscription. Après avoir dit: "Caroline, one of her daughters, is interred in the west end of this chapel, near her mother", l'auteur ajoute: "She was a good wife, a tender mother. Her death was much lamented by her family and acquaintances", ce qui s'applique à la mère. On conçoit difficilement qu'en 19 ans de ménage, Caroline Cuthbert ait pu devenir grand'mère. On serait plutôt porté à croire qu'elle est morte lors de la naissance de son dernier enfant, ou peu après.

A cette liste des enfants de Cuthbert, M. Audet ajoute un autre nom: celui de Rébecca. Rebecca Cuthbert n'était pas une fille de James Cuthbert, mais bien sa troisième femme. Elle était Américaine de naissance, et les legs que lui fait son mari sont à la condition qu'elle n'ira pas demeurer aux Etats-Unis ou ailleurs (veut-il dire hors de Berthier?). On sait d'elle peu de chose, sauf que son père était juge au New Jersey, et le nom de Gilbert et de Susan Stockton, que l'on rencontre dans certains actes de l'état civil concernant les Cuthbert, pourraient bien être ceux de son frère et de sa sœur. Le 28 juin 1790, une négresse à son service faisait baptiser, sous le nom de Rose, une fille 
de quatre mois. Le 14 octobre 1795, son mari donnait à Rebecca une procuration pour le représenter, ainsi qu'Alexander Cairns, dans les actes concernant la seigneurie, mais les actes que nous avons vus sont signés de Cairns seul. Le 30 novembre 1803, sous le nom de Rebecca Stockton, veuve, elle épouse John Kay, "housewright", de Berthier. Les témoins sont des inconnus. Le 23 juillet 1807, la Gazette de Québec annonçait la vente de leurs biens par le shérif. Enfin, elle mourut a Berthier le 14 août 1811, âgée de 69 ans, et fut inhumée le lendemain. Aucun Cuthbert n'assistait à la cérémonie. Son mari était prédécédé.

J'ai ajourné mon étude sur James Cuthbert, fils, qui eut une carrière remarquable. Si Dieu me prête vie, je ferai sa biographie pour le deuxième parlement du Bas-Canada. Pour le moment, je me contenterai de celles de l'abbé Moreau, de F.-J. Audet, du R. Père Lejeune, de W.S. Wallace et de Gustave Turcotte, toutes assez courtes, et n'y ajouterai que quelques mots.

James Cuthbert abjura le protestantisme en 1787 et persista dans ses croyances catholiques, ce qui ne plaisait pas à son père. Il fut même question d'un procès entre le père et le fils. Le père et ce fils semblent s'être réconciliés, car le père lui donne plus tard une procuration.

Quoique catholique, c'est devant le ministre anglican qu'il épousait, le 5 janvier 1804, Marie Claire Fleury Fraser, catholique, elle aussi, fille du juge John Fraser et de Marie-Anne Fleury Deschambault, après contrat de mariage passé devant Beek notaire, francmaçon notoire. Elle mourut le 11 janvier 1801 à l'Hôtel-Dieu de Montréal, après avoir fait appeler, pour lui administrer les derniers sacrements, le curé Pouget, de Berthier. Son corps, inhumé à Montréal, fut transporté à Berthier. Ils ne paraissent pas avoir eu d'enfants ${ }^{8}$.

Le 22 juin 1814, en l'église de St-Cuthbert, il épousait, après dispense du second degré de consanguinité et contrat de mariage passé devant McBean, notaire, Marie-Louise-Amable Cairns, née à Berthier, le 31 décembre 1793, fille d'Alexander Cairns, agent de la seigneurie, et de Marie Bergin (ou Berger), originaire de Québec. Elle avait trois scurs: Elizabeth, née à Berthier le 18 mars 1794, épousa en premières

8. P.-G. Roy, Les juges de la province de Québec (Québec, 1932), 223. 
noces Louis-Marie Raphael Barbier. Celui-ci mourut a Berthier, où il fut inhumé le $1^{\text {er }}$ mai 1852 . Le 4 avril suivant, elle épousait un autre médecin, Guillaume Sylvain de Bonald, natif d'Aurillac, France, beaucoup plus jeune qu'elle. Elle mourut le 25 avril 1879. Son mari épousa ensuite une fille du journaliste Etienne Parent, veuve du journaliste Evariste Gélinas. Ils vinrent vivre à Montréal, rue Craig, en face du jardin Viger.

Une demoiselle Jeanne Cairns fut inhumée à Berthier le 7 avril 1836, âgée de 40 ans, et par conséquent née en 1796. Les noms de ses parents n'apparajssent pas au registre.

Une autre sœur, Catherine, devint religieuse hospitalière sous le nom de Sœur Sainte-Agnès. Nous la perdons de vue après 1824.

Enfin, madame Cuthbert eut une autre sœur, Geneviève, qui fut marraine de deux enfants de Cuthbert: Anne-Louise-Agnès, baptisée le 10 octobre 1805, et le 6 février 1824, elle l'était de Sophie-Amélie Cuthbert. Elle était célibataire à cette époque. Une Geneviève Cairns, veuve de Daniel Morrison, mourut le 25 avril 1825. C'était la sœur de Catherine Cairns, deuxième épouse de James Cuthbert, père 9.

James Cuthbert paraît avoir dissipé ses biens, et, à la mort de M. François Baby, en 1823, il sollicitait le poste de grand-voyer du district de Québec. A cette fin, il demanda l'appui de Mgr Plessis, qui s'en lava les mains, et, contrairement à ce qu'ont dit certains historiens, il ne parait pas avoir obtenu le poste. Il s'adressa aussi en vain a $\mathbf{M g r}$ Lartigue pour se procurer des fonds. Il mourut à Berthier le 4 mars 1849 , dans sa quatre-vingtième année. Sa femme lui survécut jusqu'au 25 juillet 1878, mourant à l'âge de 90 ans. Tous deux furent inhumés dans le cimetière de Berthier. Ils avaient eu treize enfants, dont $M$. Gérard Malchelosse a relevé la généalogie, encore inédite.

Nous croyons qu'il reste encore deux descendants de James Cuthbert portant son nom patronymique: Cuthbert Cuthbert, fils d'Albert Edward Ross Cuthbert et de Charlotte Allison Nye et petit-fils d'Edward Octavian Agapit Cuthbert et de Mary Bostwick, dont il a mercie.

9. Je dois ces renseignements à madame Florence Fernet-Martel et je l'en re- 
été question plus haut. Marié deux fois, il n'a pas de postérité. Sa sœur, Margaret, née à Régina, Saskatchewan, habite New-York, où elle est attachée à l'American Broadcasting Corporation.

Je laisse à de plus érudits le soin de compléter ces notes.

Juge Edouard FABRE-SURveYer, Montréal

Note de la rédaction - Faute d'espace, nous n'avons pu publier, en cette livraison, plusieurs articles dont l'un de M. G. F. G. Stanley et un autre de M. Gabriel Debien. Nous nous en excusons auprès de nos collaborateurs. 